\title{
An Integrated BAC and Genome Sequence Physical Map of Phytophthora sojae
}

\author{
Xuemin Zhang, ${ }^{1}$ Chantel Scheuring, ${ }^{2}$ Sucheta Tripathy, ${ }^{1}$ Zhanyou Xu, ${ }^{2}$ Chengcang Wu, ${ }^{2}$ Angela Ko, ${ }^{1}$ \\ S. Ken Tian, ${ }^{1}$ Felipe Arredondo, ${ }^{1}$ Mi-Kyung Lee, ${ }^{2}$ Felipe A. Santos, ${ }^{2}$ Rays H.Y. Jiang, ${ }^{1}$ Hong-Bin Zhang, ${ }^{2}$ \\ and Brett M. Tyler ${ }^{1}$ \\ ${ }^{1}$ Virginia Bioinformatics Institute, Virginia Polytechnic Institute and State University, Blacksburg, 24061-0477, U.S.A.; \\ ${ }^{2}$ Department of Soil and Crop Sciences, Texas A\&M University, College Station 77843-2474, U.S.A.
}

Submitted 17 May 2006. Accepted 24 July 2006.

\begin{abstract}
Phytophthora spp. are serious pathogens that threaten numerous cultivated crops, trees, and natural vegetation worldwide. The soybean pathogen $P$. sojae has been developed as a model oomycete. Here, we report a bacterial artificial chromosome (BAC)-based, integrated physical map of the $P$. sojae genome. We constructed two BAC libraries, digested 8,681 BACs with seven restriction enzymes, end labeled the digested fragments with four dyes, and analyzed them with capillary electrophoresis. Fifteen data sets were constructed from the fingerprints, using individual dyes and all possible combinations, and were evaluated for contig assembly. In all, 257 contigs were assembled from the XhoI data set, collectively spanning approximately $132 \mathrm{Mb}$ in physical length. The BAC contigs were integrated with the draft genome sequence of $P$. sojae by end sequencing a total of 1,440 BACs that formed a minimal tiling path. This enabled the 257 contigs of the BAC map to be merged with 207 sequence scaffolds to form an integrated map consisting of 79 superscaffolds. The map represents the first genome-wide physical map of a Phytophthora sp. and provides a valuable resource for genomics and molecular biology research in $P$. sojae and other Phytophthora spp. In one illustration of this value, we have placed the $\mathbf{3 5 0}$ members of a superfamily of putative pathogenicity effector genes onto the map, revealing extensive clustering of these genes.
\end{abstract}

Additional keywords: BAC end sequencing (BES), BAC fingerprinting.

Phytophthora sojae (Kaufmann \& Gerdemann) is a destructive oomycete pathogen of soybean that infects the roots and stems and causes worldwide annual losses of $\$ 1$ to 2 billion, including \$200 million in the upper-Midwestern United States. Oomycetes, including $P$. sojae, are evolutionarily distant from "true" fungi and belong to the kingdom Stramenopila. Thus, oomycetes must have acquired mechanisms for pathogenicity on plants independently of pathogenic fungi, by convergent evolution. Because oomycetes are not fungi, they remain unaffected by many fungicidal control agents. Furthermore, many

Corresponding author: B. M. Tyler; Telephone: (540) 231-7318; Fax: (540) 231-2606; E-mail: bmtyler@vt.edu

$\mathrm{X}$. Zhang and C. Scheuring contributed equally to this work.

* The $\boldsymbol{e}$-Xtra logo stands for "electronic extra" and indicates the HTML abstract available on-line contains supplemental material not included in the print edition. Four additional tables appear on-line. oomycetes appear to have extraordinary genetic flexibility that enables them to adapt rapidly to both chemical control measures and genetic resistance of plant hosts.

The kingdom Stramenopila also includes organisms such as diatoms, chrysophytes, and brown algae that are secondarily photosynthetic and have acquired chloroplasts from a red algal endosymbiont. Analysis of the $P$. sojae genome sequence revealed many genes that appear to have originated from a red alga (Tyler et al. 2006), which implies that the ancestor of the Stramenopiles was already secondarily photosynthetic. Thus, the genome of $P$. sojae is of considerable evolutionary interest.

The destructiveness of oomycete pathogens, the difficulty in controlling them, and the evolutionary interest of Phytophthora spp. have led to considerable efforts to develop molecular genetic and genomic tools for this group of pathogens. The interactions of several oomycete pathogens, including $P$. sojae, with their host plants are governed by avirulence (Avr) genes (Tyler 2001, 2002). These genes encode proteins that likely contribute to the virulence of the pathogen but prevent infection when the host plant contains a resistance gene that recognizes the Avr gene product. Detailed genetic maps have been constructed for $P$. sojae that include 11 of the $13 P$. sojae Avr genes, primarily using molecular markers (May et al. 2002; Whisson et al. 1995). The $A v r$ gene $A v r l b-1$ has been cloned by map-based approaches (Shan et al. 2004), and Avrla (MacGregor et al. 2002) and Avr4/6 (Whisson et al. 2004) have been localized to small genetic and physical intervals. Avr $1 b-1$ encodes a small secreted protein that appears to enter the soybean host cells (Shan et al. 2004). Over 30,000 P. sojae expressed sequence tags have been generated and made available to the public (Qutob et al. 2000; Torto-Alalibo et al. in press). In addition, a draft shotgun sequence of the $P$. sojae genome has been completed that reveals an estimated genome size of $95 \mathrm{Mb}$ (Tyler et al. 2006). The genome sequence revealed the presence of a diverse superfamily of 350 genes with sequence similarity to Avrlb-1, termed Avh genes (Tyler et al. 2006). This superfamily shares with Avrlb-1 and three other cloned oomycete avirulence genes two motifs near the N-terminus, termed RXLR and dEER (Birch et al. 2006; Rehmany et al. 2005). The RXLR motif may be involved in entry of the Avr and related proteins into the host plant cell (Bhattacharjee et al. 2006; Birch et al. 2006; Rehmany et al. 2005).

Genome physical maps generated from large-insert library clones have proven useful for genome sequence assembly, sequence gap closure, map-based cloning, and comparative genomics. Therefore, physical maps have been constructed for several species, including human (International Human Genome Mapping Consortium 2001), mouse (Gregory et al. 2002), 
Drosophila melanogaster (Hoskins et al. 2000), Arabidopsis thaliana (Chang et al. 2001; Mozo et al. 1999), rice (Tao et al. 2001), chicken (Ren et al. 2003; Wallis et al. 2004), Penicillium spp. (Xu et al. 2005), soybean (Wu et al. 2004a), and maize (Nelson et al. 2005). Nevertheless, a genome-wide physical map has been unavailable for $P$. sojae despite its wide use as a model species for genomic and evolutionary studies of Phytophthora spp. Several methods have been developed for constructing physical maps from large-insert DNA clones by fingerprint analysis (Wu et al. 2005; Xu et al. 2004). In this study, we developed a bacterial artificial chromosome (BAC)based physical map of $P$. sojae using a multiplex, fluorescence-labeled, capillary electrophoresis-based fingerprinting method. The BAC physical map was verified and further refined by comparison with the existing whole-genome shotgun sequence of the species; in turn, this process has facilitated the assembly of the genome sequence.

\section{RESULTS}

\section{BAC library construction.}

Two BAC libraries were used for the physical map: an older library of 14,592 clones generated in 1994 by partial digestions with HindIII and a new library containing 7,680 clones constructed with BamHI for P. sojae. The DNA of 24 random clones was extracted, digested with NotI, and analyzed on a pulsedfield agarose gel. The average insert size of the clones-confirmed by BAC end sequencing (BES) alignment along the shotgun sequence scaffolds-was estimated to be $55 \mathrm{~kb}$ for the older HindIII library and $122 \mathrm{~kb}$ for the new BamHI library. Given an estimated $P$. sojae genome size of $95 \mathrm{Mb}$ (Tyler et al. 2006), the coverages of the libraries were 8.4 and 9.9 times that of the $P$. sojae genome, respectively. The combination of the two BAC libraries increased genome coverage and reduced the number of contig gaps in the physical map because of the use of different restriction enzymes for construction of the two libraries.

\section{BAC fingerprinting.}

In all, 7,680 clones from the HindIII library and 6,912 clones from the BamHI library were fingerprinted to develop the BAC map of the $P$. sojae genome. Of these clones, 11,505 yielded clear and sharp fingerprints with a sufficient number of bands to yield resolution (Fig. 1), whereas the other clones were insert empty, gave five or fewer bands, or failed in fingerprinting. The bands of each clone ranging in size from 35 to 500 bases were used for contig assembly. Each clone fingerprint consisted of 6 to 120 fragments for a single color, depending on the insert size and restriction site frequency for the relevant enzymes in the clone. The 11,505 clones were estimated to be equivalent to 10.5 times the coverage of the $P$. sojae genome, which, according to previous physical mapping studies (Chang et al. 2001; Ren et al. 2003; Tao et al. 2001; Wu et al. 2004b), should be a large enough number of clones to adequately represent the genome.

\section{BAC contig assembly.}

Using the FingerPrinted Contig (FPC) program (Soderlund et al. 1997, 2000), we attempted to assemble the physical map of the $P$. sojae genome from the 15 label-color datasets (discussed below) (Xu et al. 2005) generated from the 11,505 fingerprints. According to $\mathrm{Xu}$ and associates (2004), we modified each original band size by a factor of 10 to convert them into integers. The bands of each BAC fingerprint ranging from 35 to 500 bases in size were collected for contig assembly and $(500-35) \times 10=$ 4,650 were used as the gel length setting for one-color datasets, 9,300 for two-color datasets, 13,950 for three-color datasets, and 18,600 for four-color datasets, respectively. Automatic contig assembly, verification, editing, and merging were processed as described previously (Ren et al. 2003; Xu et al. 2005). Initial contigs were assembled at a fixed tolerance of 5 and a cutoff value of 1e-30. Each contig was verified by use of its fingerprint pattern. Chimeric contigs were split and contigs with five or more questionable clones (Qs) were rearranged at lower cutoff values using the Dqer function of the FPC program (Ren et al. 2003). The neighboring contigs were merged and singletons were added to appropriate contigs by gradually increasing to $1 \mathrm{e}-$ 20 , 1e-18, 1e-15, and 1e-12, as suggested by the FPC program and justified with their fingerprints.

The dataset generated with $\mathrm{XhoI}$ and end labeled in red had an average of 35.3 unique bands per clone and was found to yield the largest contigs with the fewest questionable clones. This result was consistent with the results of $\mathrm{Xu}$ and associates (2004, 2005). The physical map assembled from the red dataset consisted of 257 contigs of 8,681 clones and 2,822 singletons. Further analysis showed that a vast majority of the singleton clones were from the smaller-insert HindIII library, as expected given the shorter lengths of these clones. The largest contig of the map (ctg32) comprised 147 clones and spanned approximately $2.27 \mathrm{Mb}$ in physical length. The BAC physical map is summarized in Table 1 . The 257 contigs collectively contained a total of 53,727 unique bands and were estimated to span $132 \mathrm{Mb}$ in physical length. This number was larger than the $95-\mathrm{Mb}$ estimated genome size of $P$. sojae by $39 \%$ and suggests that overlaps likely existed among a large number of neighboring contig pairs.

\section{Integration of the BAC physical map with the whole-genome shotgun sequence assembly.}

To further verify and refine the physical map, we used FPC v. 6.2 to select 1,440 clones that constituted a minimum tiling path of the physical map, and we subjected the clones to BES. The clones account for $16.6 \%$ of the 8,681 clones in the physical map. Of the 2,880 BES reads, 1,933 were successfully aligned to the genome sequence scaffolds. The BAC end sequences have been submitted to GenBank's dbGSS database (accession numbers DX577376 to DX579308; dbGSS IDs 14551368 to 14553300$)$. The BES matches to the whole-genome shotgun sequence assembly were sorted first by scaffold number and then by coordinates in the scaffolds by ascending order. This analysis allowed the original 257 contigs to be merged into 79 superscaffolds. The merging of the superscaffolds is summarized in Table 2. Superscaffolds 1 to 60 contain almost all the sequence scaffolds larger than $50 \mathrm{~kb}$. Superscaffolds 61 to 79 contain the BAC contigs that do not have BES links to any sequence scaffolds. The latter group of contigs had only one to two sequenced minimum tiling path clones each, and the clones' end sequence reads either failed or lacked perfect matches to the genome sequence. The alignment of the physical map along the whole-genome shotgun sequence assembly also facilitated the shotgun sequence contig extension and gap closure processes. There were 106 single BAC clones whose end sequences could link two adjacent sequence scaffolds and condense 113 sequence scaffolds into 41 superscaffolds that encompassed $38 \mathrm{Mb}$ of sequence. Altogether, 207 sequence scaffolds were joined by single BACs or by BAC contigs to create 60 superscaffolds. The merged physical map containing the 60 superscaffolds is diagrammed in Figure 2.

\section{Map quality.}

To test the accuracy of the map, we compared the order of clones of contig 11 with their corresponding sequence scaffolds. Contig 11 was aligned to scaffolds $37,60,169$, and 1,769 in superscaffold 21 (Table 3). The sequenced clone orders in contig 11 and in scaffolds 37, 60, 169, and 1,769 of superscaffold 21 were highly consistent. Approximately $90 \%$ of the clones 
showed the same order in contig 11 and the four scaffolds named above. The order of the remaining clones that were aligned to scaffold 169 was not determined because they had only one successful end sequence each on the scaffold. Similar results were obtained for the alignment between other contigs and scaffolds. These results strongly suggest that the contig map is highly reliable.

To facilitate the public use of the physical map and database, WebFPC (Pampanwar et al. 2005) was installed to enable viewing the contigs of the FPC database with web browsers. An example is shown in Figure 3. The BAC physical map and the FPC file of the BAC fingerprints and contigs are available at the web site of the Virginia Bioinformatics Institute Microbial Database.

\section{Placement of Avh genes on the integrated map.}

In order to examine the genome-wide distribution of $P$. sojae $A v h$ genes, the positions of the genes in the genome sequence scaffolds were used to locate the positions of the genes on the integrated physical map together with the positions of the Avrlb-1, Avrla, and Avr4/6 genes (Fig. 2). The Avh genes show extensive clustering. Nine superscaffolds $(2,7,16,17,20,21$,
28, 29, and 46) contain 189 or $54 \%$ of all the Avh genes. Some short regions contain very dense clusters of $A v h$ genes; for example, on scaffolds 16, 29, and 46. In other regions, such as superscaffolds 2 and 7, there are numerous Avh genes spread throughout. In contrast, several large regions (e.g., on superscaffolds 1, 3, 13, 26, and 27) are almost devoid of Avh genes. $A v r l b-1$ is located within superscaffold 7, which has $26 A v h$ genes. Avr4/6 also is located on superscaffold 6, which has a moderate number of Avh genes $(n=8)$. Avrla is located near a cluster of five $A v h$ genes at the end of superscaffold 13, but there are no other $A v h$ genes on that superscaffold. It is not known yet if any of the Avh genes near Avrla and Avr4/6 correspond to those Avr genes.

\section{DISCUSSION}

\section{Fingerprinting method.}

The fingerprinting method we employed in this study has been demonstrated to provide high-throughput and high-information content. The 15 data sets generated simultaneously by one run of capillary electrophoresis provide extensive informa-

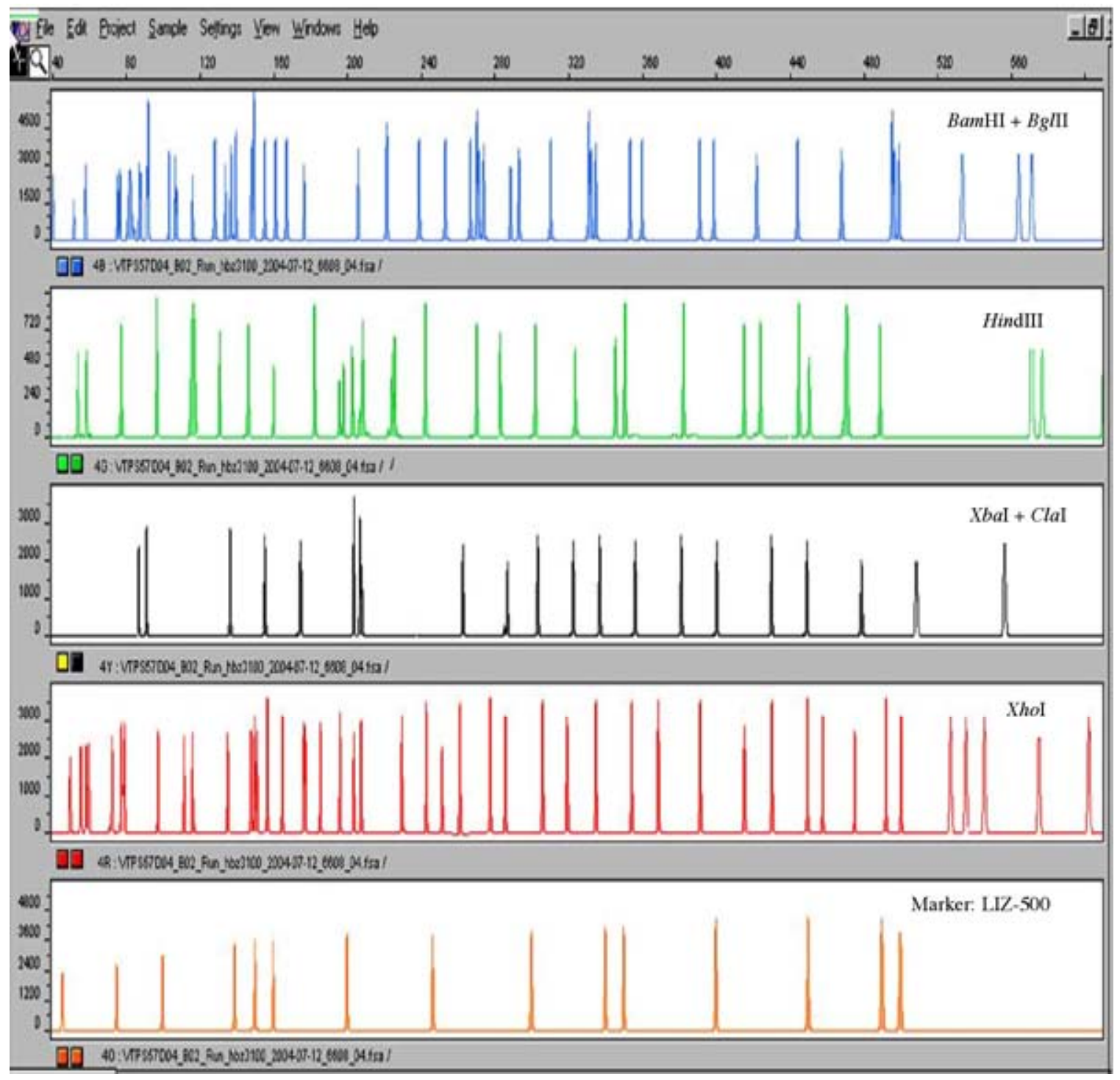

Fig. 1. Example of fingerprints of a bacterial artificial chromosome (BAC) clone (57D04). The five rows of peaks from top to bottom indicate the BAC DNA fragments with labeled ends of BamHI + BglII (blue), HindIII (green), XbaI + ClaI (black), and XhoI (red) and internal marker LIZ-500 (orange) detected by ABI 3100 DNA analyzer (Applied Biosystems, Foster City, CA, U.S.A.) in the range from 35 to 500 bases. The yellow-labeled $X b a \mathrm{I}+C l a \mathrm{I}$ fragments are represented by black by the GeneScan software (Applied Biosystems). 
tion for contig assembly. To obtain the highest quality, we tested several enzymes to find the best enzyme combination to generate a particular number of evenly distributed fragments. The best data set was used to assemble the physical map of $P$. sojae; however, if necessary, other data sets could be used to help verify potential merges or break falsely overlapping contigs. In this study, the data set generated by XhoI produced the largest and most accurate contigs and, therefore, was chosen for the physical map construction.

\section{Integration of the physical map with the sequence assembly.}

The combined strategy provides a good mechanism for reciprocal checking of the errors in each assembly. By integrating the physical map with the shotgun sequence assembly, we successfully merged or joined many scaffolds and contigs. For example, from the alignment of the $\mathrm{BAC}$ end sequences of scaffold 2, we easily could conclude that contigs 1,017, 164, and 1,346 are tandem-overlapping contigs and can be merged into a larger contig. We also found that some scaffolds were either overlapping or assembled incorrectly. For example, scaffolds 6 and 28 were joined by clone 56N24. If there was no overlap between these two scaffolds, the insert size of 56N24 must be over $1.6 \mathrm{Mb}$, which is unlikely. Therefore, we concluded that there probably is a sequence assembly error in these sequence scaffolds. Similarly, contig 1,769 probably is buried in or overlapped by scaffold 37 because of the deduced oversized insert (over $328 \mathrm{~kb}$ ) of clone 58G07. Inconsistencies like these can be resolved easily by the complete sequencing of the relevant BAC clones. The BAC end alignment also provides the necessary linking of information between BAC contigs and scaffolds which is necessary to anchor, order, and orient them to the genetic map of $P$. sojae. The BAC physical map is accessible on the web with WebFPC software (Pampanwar et al. 2005), which allows users to browse quickly through clone and contig information with a web browser instead of installing FPC software on their own computers and downloading the entire set of fingerprinting data.

We attempted to use in silico digestion to anchor the sequence scaffolds into the BAC physical map using methods similar to ones previously described (Engler et. al. 2003), but we were unsuccessful. We performed in silico digestion with the same enzymes used for our fingerprinting on some completely se-

Table 1. Attributes of the Phytophthora sojae bacterial artificial chromosome (BAC) physical map assembly

\begin{tabular}{lc}
\hline Attribute & Statistic \\
\hline Clones fingerprinted & 14,592 \\
Clones used in the map assembly & 11,505 \\
Clones assembled into contigs & 8,681 \\
Singletons & 2,822 \\
Total number of unique bands & 53,727 \\
Average bands per clone & 35.3 \\
Total number of contigs & 257 \\
Estimated physical length of the contigs & $132 \mathrm{Mb}^{\mathrm{a}}$ \\
Total number of superscaffolds & 79 \\
Estimated physical length of the superscaffolds & $>85 \mathrm{Mb}^{\mathrm{b}}$ \\
\hline
\end{tabular}

${ }^{\mathrm{a}}$ Physical map length was estimated by multiplying the total number of unique bands by the average physical length represented by each unique band. Each unique band was estimated to represent an average of 2.46 $\mathrm{kb}$, calculated by dividing the average insert size of the BAC clones from the BamHI and HindIII libraries $(87 \mathrm{~kb}$ ) by the average band number per clone (35.3).

${ }^{b}$ Physical length of the superscaffolds was estimated from the sum of the DNA sequences contained in the superscaffolds. This is an underestimate because gaps between scaffolds were not allowed for, and because superscaffolds 61 to 79 were not included in the calculation because no sequence scaffolds matched them. quenced BAC clones and on some BAC clone sequences inferred from BAC end-alignment results. The in silico fingerprints of these BACs could be assembled correctly into contigs. However, FPC could not correctly co-assemble the in silico fingerprints together with the experimental fingerprints even though we increased the cutoff to 1e-6 and the tolerance to 60 (6 bp). There are two possible reasons for this. One is that, for many fragments, there were some discrepancies between the predicted and measured sizes due to base composition effects. The deviation probably exceeded the tolerance of our FPC assembly (set at $1 \mathrm{bp}$ ), as has been observed in other studies (Ding et. al. 1999; Luo et. al. 2003). Another reason may be because of the sequence gaps in the deduced BAC insert sequences (represented by a series of "N"s in the FASTA sequence files). Recently, Nelson and associates (2005) attempted to develop a method to solve this problem, which would greatly facilitate integration between the physical map generated with automated sequencers and genome sequence contigs. Due to the difficulty of anchoring scaffolds by in silico digestion, we anchored the BAC contigs to the sequence scaffolds by sequencing the ends of a set of minimum tiling path clones. This approach enabled us to merge the 257 FPC BAC contigs into 79 superscaffolds.

\section{The map: Length, quality, utility, and smaller- and larger-insert clones.}

We compared the FPC contigs assembled with fingerprints from the smaller HindIII clones alone, the larger BamHI clones alone, and HindIII and BamHI clones together. The contigs assembled with the large-insert BamHI library showed the largest and most accurate map at different cutoffs, and the combined library data showed a moderate-quality map. The HindIII library showed poor quality in all the assemblies (data not shown); however, because the HindIII library has been used widely in $P$. sojae research for several years, we finally chose to use the combined libraries so the HindIII library-related information could be integrated into the final physical map.

\section{Distribution of $A v r$ gene homologs throughout the $P$. sojae genome.}

Placement of 350 Avh genes homologous to cloned oomycete avirulence genes revealed extensive heterogeneity in the distribution of the genes. A large proportion of the genes were concentrated in a small number of superscaffolds. Furthermore, there were many regions of the genome that were devoid of $A v h$ genes. Some of the clusters of Avh genes were concentrated in very short regions, suggesting that they had arisen by local sequence duplications. However, in other regions, such as superscaffolds 2, 6, and 7, the $A v h$ genes were distributed more uniformly. In superscaffold 7, $25 A v h$ genes plus $A v r l b-1$ are spread throughout a region of approximately $2 \mathrm{Mb}$, with an average spacing of approximately $80 \mathrm{~kb}$. The genes in this superscaffold are not unusually similar to one another (R. Jiang and B. M. Tyler, unpublished); therefore, it is unclear whether their

Table 2. Distribution of clones in bacterial artificial chromosome (BAC) contigs and superscaffolds

\begin{tabular}{lcc}
\hline $\begin{array}{l}\text { Clone numbers in contigs or } \\
\text { superscaffolds }\end{array}$ & $\begin{array}{c}\text { Number of BAC } \\
\text { contigs }\end{array}$ & $\begin{array}{c}\text { Number of } \\
\text { superscaffolds }\end{array}$ \\
\hline$>200$ clones $^{\mathrm{a}}$ & 1 & 19 \\
$100-199$ clones & 10 & 8 \\
$80-99$ clones & 9 & 5 \\
$60-79$ clones & 24 & 1 \\
$40-59$ clones & 34 & 8 \\
$20-39$ clones & 61 & 7 \\
$2-19$ clones & 118 & 31 \\
\hline
\end{tabular}

a The largest BAC contig and superscaffold contained 257 and 633 clones, respectively. 
co-location represents a recent common origin. Comparison of the locations of $A v h$ genes in the $P$. sojae and $P$. ramorum genomes revealed that $A v h$ genes have an unusually low frequency of gene colinearity (synteny); only 18 of the $350 A v h$ genes in each genome had syntenic locations (Jiang et al. 2006; Tyler et al. 2006). Detailed comparison of syntenic regions of the $P$. sojae and $P$. ramorum genomes revealed that Avh genes were commonly located in segments of DNA sequence that disrupted an otherwise continuous region of synteny (Jiang et al. 2006; Tyler et al. 2006). For example, $A v r 1 b-1$ is located within a synteny-disrupting region that includes a 10-kb indel, two translocations, and an inversion
(Jiang et al. 2006; Tyler et al. 2006). These observations suggest that Avh genes may be associated with sites of elevated genome fluidity. The presence of extended regions such as superscaffold 7 that contain a high concentration of $A v h$ genes suggests, in turn, that there may be larger regions of the genome that have elevated genome fluidity. It will be interesting to discover whether regions that have high concentrations of $A v h$ genes are located near the ends of chromosomes; in other organisms, the tips of chromosomes are sites of elevated fluidity and of concentrations of virulence genes (Eichler and Sankoff 2003; Farman and Kim 2005; Freitas-Junior et al. 2000; Liu et al. 1985).
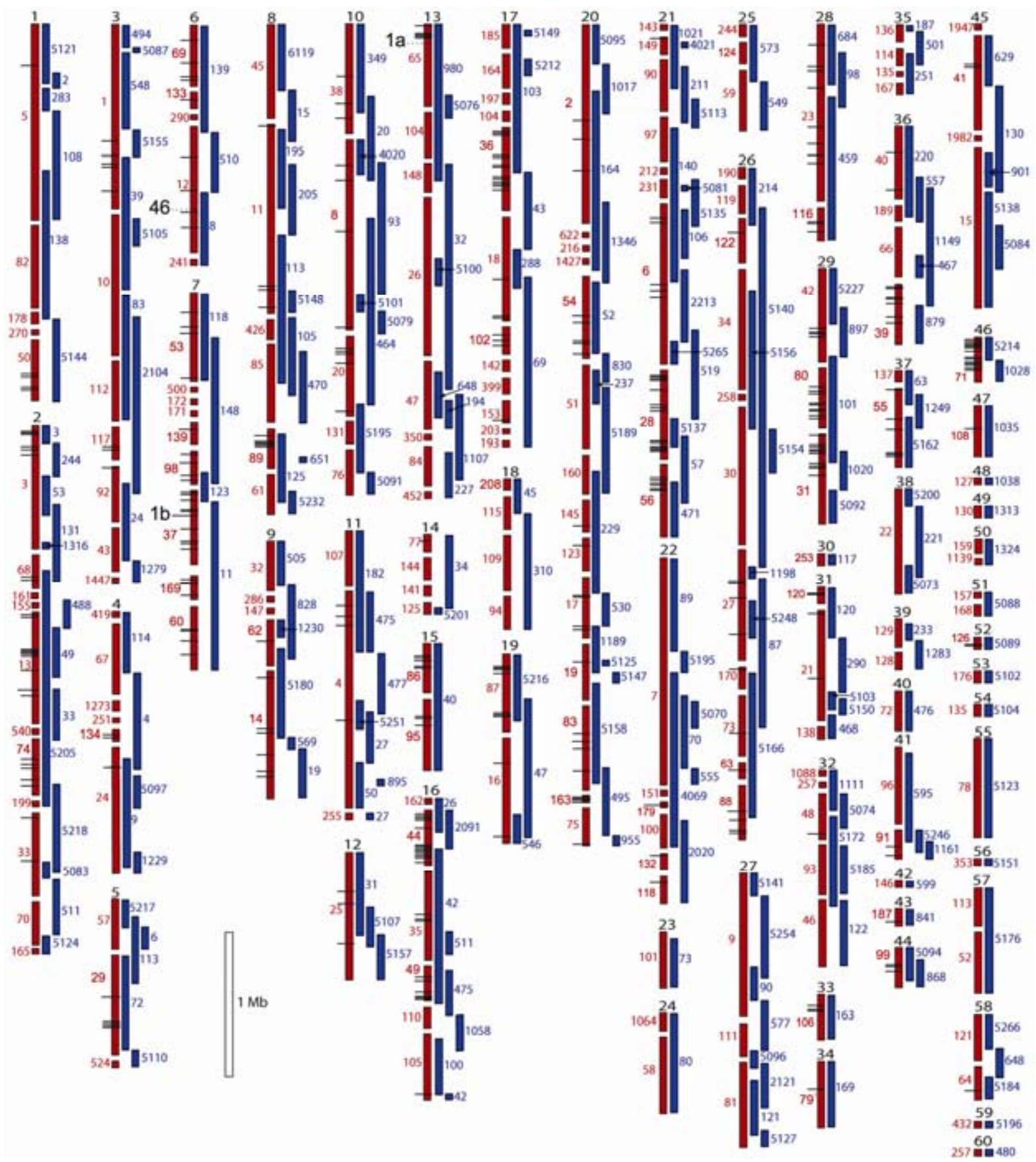

Fig. 2. Sequence scaffolds of Phytophthora sojae integrated with the physical map. The scale is approximate. Red bars and numbers indicate sequence scaffolds. Blue bars and numbers indicate bacterial artificial chromosome contigs assembled by fingerprinting. Large black numbers indicate superscaffold numbers. Horizontal black lines indicate positions of Avh genes in each sequence scaffold. "1a," "1b," and " 46 " indicate the published positions of P. sojae avirulence genes Avrla (MacGregor et al. 2002), Avrlb-1 (Shan et al. 2004), and Avr416 (Whisson et al. 2004). It is not yet known whether the Avrla and Avr4/6 genes correspond to Avh genes; therefore, their positions are indicated with dotted lines. Superscaffolds 61 to 79 are not shown because they had no sequence matches. 


\section{MATERIALS AND METHODS}

Strain and cloning vector.

P. sojae strain P6497 (race 2) (Förster et al. 1994) was used as the DNA source for both libraries. Vector pBeloBAC11 (Shizuya et al. 1992) was used for the HindIII library, and vector pECBAC1 (Frijters et al. 1997) was used for the BamHI library. Strain P6497 also was used for the whole-genome shotgun sequencing of the species (Tyler et al. 2006).

\section{High molecular weight DNA preparation.}

One-week-old $P$. sojae mycelia grown in clarified V8 medium (Erwin and Ribeiro 1996) were harvested and dried by vacuum filtration and then resuspended and incubated in isotonic buffer $\left(0.7 \mathrm{M} \mathrm{KCl}, 50 \mathrm{mM} \mathrm{KH} \mathrm{PO}_{4}, \mathrm{pH} 5.8\right)$ containing glucanex, a lytic cell-wall-degrading enzyme. Undigested mycelia and cellular debris were separated by centrifugation $\left(5,000 \times g, 4^{\circ} \mathrm{C}, 20 \mathrm{~min}\right)$, and protoplasts were washed with STC (1.2 M sorbitol, $10 \mathrm{mM} \mathrm{CaCl}_{2}$, and $10 \mathrm{mM}$ Tris- $\mathrm{HCl}, \mathrm{pH}$ 7.5) and stored in STC at $-80^{\circ} \mathrm{C}$ before use.

Frozen $P$. sojae protoplasts $\left(5 \times 10^{9}\right.$ protoplasts $\left.\mathrm{ml}^{-1}\right)$ were thawed on ice and then centrifuged at $5,000 \times g$ at $4^{\circ} \mathrm{C}$ for 20 min. Protoplasts were washed in SCE buffer (1 M sorbitol, 20 $\mathrm{mM}$ sodium citrate, $10 \mathrm{mM}$ EDTA, $\mathrm{pH} \mathrm{5.8,} 10 \mathrm{mM} \mathrm{CaCl}_{2}$ ) and then centrifuged again at $5,000 \times g$ at $4^{\circ} \mathrm{C}$ for $15 \mathrm{~min}$. The protoplast pellet was resuspended in $1 \mathrm{ml}$ of SCE and warmed to $45^{\circ} \mathrm{C}$ in a water bath; then, $1.5 \mathrm{ml}$ of SCE with $1 \%$ low-meltingpoint agarose at $45^{\circ} \mathrm{C}$ was added to the protoplasts and gently mixed. The protoplast-agarose mixture was pipetted into a 100- $\mu l$ plug mold (Bio-Rad, Hercules, CA, U.S.A.). After the plugs solidified, nuclei were lysed and DNA was purified as described previously (Ren et al. 2005; Wu et al. 2004b; Xu et al. 2005).

\section{BAC library construction.}

A BAC library containing 14,592 clones with an average insert size of approximately $55 \mathrm{~kb}$ was constructed previously for P. sojae strain P6497 using HindIII in pBeloBAC11 (F. Arredondo and B. M. Tyler, unpublished). The library has been distributed to several laboratories that have used it as a resource for $P$. sojae genomics research. For the work presented here, 7,680 clones of this library (originally designated $1 \mathrm{~A} 1$ to $6 \mathrm{P} 24$ and 9A1 to 22P24) were re-arrayed into a 96-well format for high-throughput DNA extraction. The remaining 6,912 clones from the HindIII library (7A1 to 8P24 and 23A1 to 32P24) were found to have an unacceptably high frequency of missing inserts (approximately 50\%) and were not used for fingerprinting or end sequencing.

Considering the relatively small insert size of this library for genome physical mapping, and to improve the genome coverage of $P$. sojae, we constructed a larger-insert library with BamHI in pECBAC1 for the $P$. sojae strain in this study. The library was constructed as described previously (Ren et al. 2005; Wu et al. 2004b; Xu et al. 2005; Zhang 2000). High molecular weight DNA of $P$. sojae was partially digested with $B a m \mathrm{HI}$ and subjected to size selection by pulsed-field agarose gel electrophoresis. DNA fragments of between 100 to $300 \mathrm{~kb}$ were recovered, ligated to dephosphorylated pECBAC1 that had been digested with BamHI, and transformed into Esche-

Table 3. Comparison of bacterial artificial chromosome (BAC) order in BAC contig II inferred from fingerprint assembly and genome sequence matches

\begin{tabular}{|c|c|c|c|c|c|}
\hline \multirow[b]{2}{*}{ BAC end sequence ${ }^{b}$} & \multirow[b]{2}{*}{ FPC order ${ }^{c}$} & \multicolumn{2}{|c|}{ BES match $^{\mathbf{a}}$} & \multirow[b]{2}{*}{ Genome sequence $^{\mathrm{f}}$} & \multirow[b]{2}{*}{ Orientation of scaffold } \\
\hline & & Coordinates $^{d}$ & Orientation $^{\mathrm{e}}$ & & \\
\hline 52C13-R & 1 & $37: 511358-511979$ & Opposite & 37 & $3^{\prime}-5^{\prime}$ \\
\hline 54B12-F & 2 & $37: 440922-441595$ & Opposite & 37 & $3^{\prime}-5^{\prime}$ \\
\hline $52 \mathrm{C} 13-\mathrm{F}$ & $\ldots$ & $37: 358847-359426$ & Same & 37 & $3^{\prime}-5^{\prime}$ \\
\hline 44J21-R & 4 & $37: 332376-332853$ & Opposite & 37 & $3^{\prime}-5^{\prime}$ \\
\hline 54B12-R & $\ldots$ & $37: 331016-331625$ & Same & 37 & $3^{\prime}-5^{\prime}$ \\
\hline $58 \mathrm{G} 07-\mathrm{R} *$ & 3 & $37: 296300-296957$ & Same & 37 & $3^{\prime}-5^{\prime}$ \\
\hline 54A18-F & 5 & $37: 295081-295711$ & Opposite & 37 & $3^{\prime}-5^{\prime}$ \\
\hline $44 J 21-\mathrm{F}$ & $\ldots$ & $37: 206832-207549$ & Same & 37 & $3^{\prime}-5^{\prime}$ \\
\hline 54A18-R & $\ldots$ & $37: 129536-130127$ & Same & 37 & $3^{\prime}-5^{\prime}$ \\
\hline $41009-\mathrm{R} * *$ & 7 & $37: 125599-126237$ & Opposite & 37 & $3^{\prime}-5^{\prime}$ \\
\hline $58 \mathrm{G} 07-\mathrm{F}^{*}$ & $\ldots$ & $1,769: 2858-3322$ & Same & 1,769 & $5^{\prime}-3^{\prime}$ \\
\hline $57 J 23-\mathrm{R}$ & 8 & 169:15151-15820 & Same & 169 & $5^{\prime}-3^{\prime}$ \\
\hline 49M10-R & 6 & 169:46812-47498 & Opposite & 169 & $5^{\prime}-3^{\prime}$ \\
\hline $44 \mathrm{~N} 12-\mathrm{F}^{* * *}$ & 9 & $169: 49774-50303$ & Same & 169 & $5^{\prime}-3^{\prime}$ \\
\hline $41009-\mathrm{F}^{* *}$ & $\ldots$ & $169: 58642-59325$ & Opposite & 169 & $5^{\prime}-3^{\prime}$ \\
\hline 41019-R & 10 & 60:17494-18103 & Same & 60 & $5^{\prime}-3^{\prime}$ \\
\hline $44 \mathrm{~N} 12-\mathrm{R} * * *$ & $\ldots$ & $60: 87920-88485$ & Opposite & 60 & $5^{\prime}-3^{\prime}$ \\
\hline $49 \mathrm{C} 05-\mathrm{F}$ & 11 & $60: 97885-98615$ & Same & 60 & $5^{\prime}-3^{\prime}$ \\
\hline 58A19-R & 12 & $60: 133531-134212$ & Same & 60 & $5^{\prime}-3^{\prime}$ \\
\hline 41019-F & $\ldots$ & $60: 190441-191175$ & Opposite & 60 & $5^{\prime}-3^{\prime}$ \\
\hline $49 \mathrm{C} 05-\mathrm{R}$ & $\ldots$ & $60: 193377-194104$ & Opposite & 60 & $5^{\prime}-3^{\prime}$ \\
\hline 58A19-F & $\ldots$ & $60: 253156-253865$ & Opposite & 60 & $5^{\prime}-3^{\prime}$ \\
\hline 03K19-F & 13 & $60: 256876-257512$ & Same & 60 & $5^{\prime}-3^{\prime}$ \\
\hline 57L07-R & 14 & $60: 274389-274725$ & Same & 60 & $5^{\prime}-3^{\prime}$ \\
\hline 03K19-R & $\ldots$ & $60: 354390-354707$ & Opposite & 60 & $5^{\prime}-3^{\prime}$ \\
\hline 57L07-F & $\ldots$ & $60: 427398-427717$ & Opposite & 60 & $5^{\prime}-3^{\prime}$ \\
\hline
\end{tabular}

${ }^{\mathrm{a}} \mathrm{BES}=\mathrm{BAC}$ end sequencing.

${ }^{\mathrm{b}}$ Sequences obtained from each BAC using the M13 forward $(\mathrm{F})$ or reverse $(\mathrm{R})$ primer; *, **, and *** indicate pairs of BAC end sequences that match two different sequence scaffolds.

${ }^{c}$ Relative order of the leftmost end of each BAC in the FingerPrinted Contig (FPC) assembly of BAC contig 11.

${ }^{\mathrm{d}}$ Scaffold number (before the colon) and coordinates within the scaffold (after the colon) of each BAC end sequence match to the draft genome sequence of Phytophthora sojae.

${ }^{\mathrm{e}}$ Same $=$ the BES matched the scaffold sequence in the same arbitrary orientation as the scaffold sequence and opposite $=$ the reverse complement of the BES matched the scaffold sequence.

${ }^{\mathrm{f}}$ Genome sequence scaffold.

g Orientation of the scaffold sequence relative to the overall orientation of the aggregate sequence of the superscaffold, inferred from the relative orientations of the BAC end sequences that match two adjoining scaffolds. 
richia coli DH10B cells. The resulting white transformant clones growing on selective medium containing chloramphenicol, IPTG, and X-gal were arrayed into 384-well microplates. The numbering of the BamHI clones was started from 41A1 so that the clones could be distinguished readily from the HindIII clones (1A1 to 20D96). P. sojae BAC clones may be requested via the web site of the Oomycete Molecular Genetics Research Collaboration Network or by contacting the corresponding author.

\section{BAC DNA purification.}

Both the HindIII and BamHI BAC libraries were used for genome physical mapping. Although the HindIII library has a smaller insert size, it has been used widely in genome research of $P$. sojae. Clones of the libraries were transferred to 96-well plates with $1.2 \mathrm{ml}$ of $2 \times \mathrm{LB}$ (20 g of tryptone, $10 \mathrm{~g}$ of yeast extract, and $10 \mathrm{~g}$ of $\mathrm{NaCl}$ per liter) and chloramphenicol at $12.5 \mu \mathrm{g} / \mathrm{ml}$ in each well. The clones then were grown at $37^{\circ} \mathrm{C}$ for $24 \mathrm{~h}$ with shaking at $250 \mathrm{rpm}$. BAC DNA was purified with either the Perfectprep BAC 96 Kit (Eppendorf North America, Inc., Westbury, NY, U.S.A.) or the DNA Isolation Robotic Workstation AutoGenprep 960 (Autogen, Inc., Holliston, MA, U.S.A.). Purified DNA samples were suspended in $80 \mu \mathrm{l}$ of double-distilled water and kept in 96 -well plates at $-80^{\circ} \mathrm{C}$ before use.

\section{BAC fingerprinting.}

BAC DNA samples (35 $\mu \mathrm{l}$ each) were transferred to 96-well microtube plates, digested, and labeled with a fingerprinting kit according to $\mathrm{Xu}$ and associates (2005), with some modifications. The fingerprinting kit contained six 6-bp endonucleases (BamHI, BglII, XbaI, ClaI, HindIII, and XhoI) in order to generate sufficient numbers of bands for the smaller-insert clones, one 4-bp endonuclease (HaeIII), and the SNaPshot Multiplex Ready-Reaction Mix (Applied Biosystems, Foster City, CA, U.S.A.). The fragment ends were labeled as follows: $B a m \mathrm{HI}$ and BglII with ddGTP-dR110 (blue), XbaI and ClaI with ddCTP-dTAMRA (yellow), HindIII with ddATP-dR6G (green), and XhoI with ddTTP-dROX (red). HaeIII was used to cut the 6-bp enzyme fragments into smaller ones so the fragments could be fractionated on a capillary sequencer in a range of 35 to $500 \mathrm{bp}$. Because fragment ends created by HaeIII were blunt, they were not labeled in the reaction.

The multi-enzyme digestion and labeling reactions were carried out as follows. First, the DNA was digested with HindIII, $\mathrm{XbaI}, \mathrm{XhoI}$, and $\mathrm{Bg} l \mathrm{II}$ at $37^{\circ} \mathrm{C}$ for $2 \mathrm{~h}$, inactivated by incubation at $65^{\circ} \mathrm{C}$ for $18 \mathrm{~min}$, and cooled on ice. Next, samples were centrifuged and then were digested with BamHI, ClaI, and HaeIII and simultaneously labeled with the SNaPshot mix at $37^{\circ} \mathrm{C}$ for another $2 \mathrm{~h}$. Afterward, samples were transferred to a $65^{\circ} \mathrm{C}$ incubator for $1 \mathrm{~h}$ to stop the digestion but continue the labeling. The DNA was precipitated, washed, dried, and dissolved in a mixture of $9.8 \mu \mathrm{l}$ of Hi-Di formamide and $0.2 \mu \mathrm{l}$ of internal size marker LIZ-500 (Applied Biosystems). Samples were denatured at $95^{\circ} \mathrm{C}$ for $8 \mathrm{~min}$, incubated on ice for $8 \mathrm{~min}$, and loaded into an ABI 3100 DNA Analyzer (Applied Biosystems) fitted with $36-\mathrm{cm}$ capillaries. The raw fingerprints in the window ranging from 35 to 500 bases were collected with the default GeneScan v. 3.70 module and the ABI Prism 3100 Data Collection software v. 1.0.1.

\section{Contig assembly.}

Raw fingerprint data from the ABI 3100 DNA analyzer were edited and converted into FPC band data as described previ-

\section{WebFPC: contig_bac}



Fig. 3. Bacterial artificial chromosome (BAC) contig (Ctg11) of the Phytophthora sojae genome displayed in the WebFPC window. This contig contains 140 BAC clones, including 19 sequenced minimum tiling path clones (highlighted). Not all the sequenced clones yielded matches to the sequence assembly. The total physical length of this contig was estimated to be $1.79 \mathrm{Mb}$. 
ously (Xu et al. 2004, 2005). Each clone yielded four sets of fingerprints, and each set was labeled with a single color. Therefore, 15 datasets in total were assembled from the fingerprints: 4 single-color datasets (blue, green, yellow, and red), 6 twocolor combination datasets (blue+green, blue+yellow, blue+red, green+yellow, green+red, and yellow+red), 4 three-color combination datasets (blue+green+yellow, blue+green+red, blue+ yellow+red, and green+yellow+red), and 1 four-color combination (blue+green+yellow+red). Contigs were assembled from each of the datasets with the software FPC v. 6.2 (Soderlund et al. 1997, 2000). Contigs were assembled first at a tolerance of 5 and with a cutoff value chosen to limit the number of questionable clones to less than 5\%. The contigs then were examined manually and merged. FPC contigs were viewed with web browsers using WebFPC (Pampanwar et al. 2005), a web-based java program.

\section{BES.}

Contig assembly by FPC v. 6.2 yielded a minimal overlapping clone tiling path of the genome consisting of 1,440 BACs (16.6\% of the total clones). These clones were arrayed into 15 96-well microplates. The two ends of each BAC were sequenced by Agencourt Bioscience Corporation (Beverly, MA, U.S.A.) with the primers Universal T7 forward (5' TAA TAC GAC TCA CTA 3') and M13 reverse (5' CAG GAA ACA GCT ATG ACC $\left.3^{\prime}\right)$.

\section{Integration of BAC contig map with shotgun sequence assembly by $\mathrm{BAC}$ end sequence alignment.}

$\mathrm{BAC}$ end sequences were used as anchor tags to align BAC contigs against the $P$. sojae shotgun genome assembly (Tyler et al. 2006). Clone names, contig numbers, and coordinates of the BAC end sequences in the sequence scaffold were sorted in a spreadsheet. A link of three or more BAC end sequences to a sequence scaffold was used to align an FPC BAC contig to the sequence scaffold, provided that $60 \%$ of the contig BAC end sequences matched the order in the sequence scaffold. Aligned contigs and scaffolds were merged into superscaffolds. Contigs with fewer than three links to scaffolds were joined into superscaffolds only if the clones were at the ends of the contigs.

\section{ACKNOWLEDGMENTS}

We thank C. Soderlund at the University of Arizona for help with FPC and WebFPC software installation. Special thanks to D. Baker and M. C. Chibucos for assistance with preparation and editing of the manuscript. This work was supported by grants to B. M. Tyler from the National Research Initiative of the United States Department of Agriculture Cooperative State Research, Education, and Extension Service, grant numbers 2001-35319-14251 and 2002-35600-12747, and the United States National Science Foundation, grant numbers MCB-0242131 and EF-0130263, and by funds from the United States Department of Energy Joint Genome Institute and the Virginia Bioinformatics Institute. R. H. Y. Jiang was supported in part by a travel grant from the Technology Foundation STW of The Netherlands and fellowship NGI 050-72-404 from Netherlands Genomics Initiative.

\section{LITERATURE CITED}

Bhattacharjee, S., Hiller, N. L., Liolios, K., Win, J., Kanneganti, T. D., Young, C., Kamoun, S., and Haldar, K. 2006. The malarial host-targeting signal is conserved in the Irish potato famine pathogen. PLoS Pathog. 2:0453-0465.

Birch, P. R., Rehmany, A. P., Pritchard, L., Kamoun, S., and Beynon, J. L. 2006. Trafficking arms: Oomycete effectors enter host plant cells. Trends Microbiol. 14:8-11.

Chang, Y. L., Tao, Q., Scheuring, C., Meksem, K., and Zhang, H.-B. 2001. An integrated map of Arabidopsis thaliana for functional analysis of its genome sequence. Genetics 159:1231-1242.
Ding, Y., Johnson, M. D., Colayco, R., Chen, Y. J., Melnyk, J., Schmitt, H., and Shizuya, H. 1999. Contig assembly of bacterial artificial chromosome clones through multiplexed fluorescence-labeled fingerprinting. Genomics 56:237-246.

Eichler, E. E., and Sankoff, D. 2003. Structural dynamics of eukaryotic chromosome evolution. Science 301:793-797.

Engler, F. W., Hatfield, J., Nelson, W., and Soderlund, C. A. 2003. Locating sequence on FPC maps and selecting a minimal tiling path. Genome Res. 13:2152-2136.

Erwin, D. C., and Ribeiro O. K. 1996. Phytophthora Diseases Worldwide. The American Phytopathological Society Press, St. Paul, MN, U.S.A.

Farman, M. L., and Kim, Y.-S. 2005. Telomere hypervariability in Magnaporthe oryzae. Mol. Plant Pathol. 6:287-298.

Förster, H., Tyler, B. M., and Coffey, M. D. 1994. Phytophthora sojae races have arisen by clonal evolution and by rare outcrosses. Mol. Plant-Microbe Interact. 7:780-791.

Freitas-Junior, L. H., Bottius, E., Pirrit, L. A., Deitsch, K. W., Scheidig, C., Guinet, F., Nehrbass, U., Wellems, T. E., and Scherf, A. 2000. Frequent ectopic recombination of virulence factor genes in telomeric chromosome clusters of $P$. falciparum. Nature 407:1018-1022.

Frijters, A. C. J., Zhang, Z., Van Damme, M., Wang, G. L., Ronald, P. C., and Michelmore, R. W. 1997. Construction of a bacterial artificial chromosome library containing large EcoRI and HindIII genomic fragments of lettuce. Theor. Appl. Genet. 94:390-399.

Gregory, S. G., Sekhon, M., Schekn, J., Zhao, S., Osoegawa, K., Scott, G., Evans, R., Burridge, P., Cox, T., Fox, K., et al. 2002. A physical map of the mouse genome. Nature 418:743-750.

Hoskins, R. A., Nelson, C. R., Berman, B., Laverty, T., Geroge, R., Ciesiolka, L., Naeemuddin, M., Arenson, A., Durbin, J., David, D. et al. 2000. A BAC-based physical map of the major autosomes of Drosophila melanogaster. Science 287:2271-2274.

International Human Genome Mapping Consortium. 2001. A Physical map of the human genome. Nature 409:934-941.

Jiang, R. H. Y., Tyler, B. M., and Govers, F. 2006. Comparative analysis of Phytophthora genes encoding secreted proteins reveals conserved synteny and lineage specific gene duplications and deletions. Mol. Plant-Microbe Interact. 19:1311-1321

Liu, A. Y. C., Michels, P. A. M., Bernards, A., and Borst, P. 1985. Trypanosome variant surface glycoprotein genes expressed early in infection. J. Mol. Biol. 182:383-396.

Luo, M.-C., Thomas, C., You, F. M., Hsiao, J., Ouyang, S. C., Buell, C. R., Malandro, M., McGuire, P. E., Anderson, O. D., and Dvorak, J. 2003. High-throughput fingerprinting of bacterial artificial chromosomes using the SNaPshot labeling kit and sizing of restriction fragments by capillary electrophoresis. Genomics 82:378-389.

MacGregor, T., Bhattacharyya, M., Tyler, B.M., Bhat, R., Schmitthenner, A. F., and Gijzen, M. 2002. Genetic and physical mapping of Avrla in Phytophthora sojae. Genetics 160:949-959.

May, K. J., Whisson, S. C., Zwart, R. S., Searle, I. R., Irwing, J. A. G., Maclean, D. J., Darroll, B. J., and Drenth, A. 2002. Inheritance and mapping of eleven avirulence genes in Phytophthora sojae. Fungal Genet. Biol. 37(1):1-12.

Mozo, T., Dewar, K., Dunn, P., Ecker, J., Fischer, S., Kloska, S., Lehrach, H., Marra, M., Martienssen, R., Meier-Ewert, S., and Altmann, T. 1999. A complete BAC-based physical map of the Arabidopsis thaliana genome. Nat. Genet. 22:271-275.

Nelson, W. M., Bharti, A. K., Butler, E., Wei, F., Fuks, G., Kim, H., Wing, R. A., Messing, J., and Soderlund, C. 2005. Whole-genome validation of high-information-content fingerprinting. Plant Physiol. 139:27-38.

Pampanwar, V., Engler, F., Hatfield, J., Blundy, S., Gupta, G., and Soderlund, C. 2005. FPC Web tools for rice, maize, and distribution. Plant Physiol. 138:116-126.

Qutob, D., Hraber, P., Sobral, B., and Gijzen, M. 2000. Comparative analysis of expressed sequences in Phytophthora sojae. Plant Physiol. 123:243-253.

Rehmany, A. P., Gordon, A., Rose, L. E., Allen, R. L., Armstrong, M. R., Whisson, S. C., Kamoun, S., Tyler, B. M., Birch, P. R., and Beynon, J. L. 2005. Differential recognition of highly divergent downy mildew avirulence gene alleles by RPP1 resistance genes from two Arabidopsis lines. Plant Cell 17:1839-1850.

Ren, C., Lee, M.-K., Yan, B., Ding, K., Cox, B., Romanov, M. N., Price, J. A., Dodgson, J. B., and Zhang, H.-B. 2003. A BAC-based physical map of the chicken genome. Genome Res. 13:2754-2758.

Ren, C., Xu, Z. Y., Sun, S., Lee, M.-K., Wu, C., Scheuring, C., Santos, T. S., and Zhang, H.-B. 2005. Genomic DNA libraries and physical mapping. Pages 173-213 in: The Handbook of Plant Genome Mapping: Genetic and Physical Mapping. K. Meksem and G. Kahl, eds. Wiley-VCH Verlag GmbH, Weinheim, Germany.

Shan, W., Cao, M., Leung, D., and Tyler, B. M. 2004. The Avrlb locus of Phytophthora sojae encodes an elicitor and a regulator required for 
avirulence on soybean plants carrying resistance gene Rps $1 b$. Mol. Plant-Microbe Interact. 17:394-403.

Shizuya, H., Birren, B., Kim, U. J., Mancino, V., Slepak, T., Tachiiri, U., and Simon, M. 1992. Cloning and stable maintenance of 300-kilobasepair fragments of human DNA in Escherichia coli using an F-factorbased vector. Proc. Natl. Acad. Sci. U.S.A. 89:8794-8797.

Soderlund, C., Humphrey, S., Dunham, A., and French, L. 2000. Contigs built with fingerprints, markers, and FPC V4.7. Genome Res. 10:17721787.

Soderlund, C., Longden, I., and Mott, R. 1997. FPC: A system for building contigs from restriction fingerprinted clones. CABIOS 13:523-535.

Tao, Q.-Z., Chang, Y.-L., Wang, J., Chen, H., Schuering, C., Islam-Faridi, M. N., Wang, B., Stelly, D. M., and Zhang, H.-B. 2001. Bacterial artificial chromosome-based physical map of the rice genome constructed by restriction fingerprint analysis. Genetics 158:1711-1724.

Torto-Alalibo, T., Tripathy, S., Smith, B. M., Arredondo, F., Zhou, L., Li, H., Qutob, D., Gijzen, M., Mao, C., Sobral, B. W. S., Waugh, M. E., Mitchell, T. K., Dean, R. A., and Tyler, B. M. Expressed sequence tags from Phytophthora sojae reveal genes specific to development and infection. Mol. Plant-Microbe Interact. in press.

Tyler, B. M. 2001. Genetics and genomics of the oomycete-host interface. Trends Genet. 17:611-614.

Tyler, B. M. 2002. Molecular Basis of Recognition Between Phytophthora species and their hosts. Annu. Rev. Phytopathol. 40:137-167.

Tyler, B. M., Tripathy, S., Zhang, X., Dehal, P., Jiang, R. H. Y., Aerts, A., Arredondo, F. D., Baxter, L., Bensasson, D., Beynon, J. L., Chapman, J., Damasceno, C. M. B., Dorrance, A. E., Dou, D., Dickerman, A. W., Dubchak, I. L., Garbelotto, M., Gijzen, M., Gordon, S. G., Govers, F., Grunwald, N. J., Huang, W., Ivors, K. L., Jones, R. W., Kamoun, S., Krampis, K., Lamour, K. H., Lee, M.-K., McDonald, W. H., Medina, M., Meijer, H. J. G., Nordberg, E. K., Maclean, D. J., Ospina-Giraldo, M. D., Morris, P. F., Phuntumart, V., Putnam, N. H., Rash, S., Rose, J. K. C., Sakihama, Y., Salamov, A. A., Savidor, A., Scheuring, C. F., Smith, B. M., Sobral, B. W. S., Terry, A., Torto-Alalibo, T. A., Win, J., Xu, Z., Zhang, H., Grigoriev, I. V., Rokhsar, D. S., and Boore, J. L. 2006. Phytophthora genome sequences uncover evolutionary origins and mechanisms of pathogenesis. Science. 313:1261-1266.

Wallis, J. W., Aerts, J., Groenen, M. A. M., Crooijmans, R. P. M. A., Layman, D., Graves, T. A., Scheer, D. E., Kremitzki, C., Fedele, M. J., Mudd, N. K., et al. 2004. A physical map of the chicken genome. Nature 432:761-764.

Whisson, S. C., Basnayake, S., Maclean, D. J., Irwin, J. A., and Drenth, A. 2004. Phytophthora sojae avirulence genes Avr4 and Avr6 are located in a $24 \mathrm{~kb}$, recombination-rich region of genomic DNA. Fungal Genet. Biol. 41:62-74.
Whisson, S. C., Drenth, A., Maclean, D. J., and Irwing, J. A. G. 1995. Phy tophthora sojae avirulence genes, RAPD and RFLP markers used to construct a detailed genetic linkage map. Mol. Plant-Microbe Interact. 8:988-955.

Wu, C., Sun, S., Nimmakayala, P., Santos, F. A., Springman, R., Ding, K. J., Meksem, K., Lightfoot, D., and Zhang, H.-B. 2004a. A BAC and BIBAC-based physical map of the soybean genome. Genome Res. 14:319-326.

Wu, C., Xu, Z., and Zhang, H.-B. 2004b. DNA libraries. Pages 385-425 in: Encyclopedia of Molecular Cell Biology and Molecular Medicine, 2nd ed., vol. 3. R. A. Meyers, eds. Wiley-VCH Verlag GmbH, Weinheim, Germany.

Wu, C., Sun, S., Lee, M.-K., Xu, Z. Y., Ren, C., and Zhang, H.-B. 2005. Whole genome physical mapping: An overview on methods for DNA fingerprinting. Pages 257-284 in: The Handbook of Plant Genome Mapping: Genetic and Physical Mapping. K. Meksem and G. Kahl, eds. Wiley-VCH Verlag GmbH, Weinheim, Germany.

Xu, Z., Sun, S., Covaleda, L., Ding, K., Zhang, A., Wu, C., Scheuring, C. and Zhang, H.-B. 2004. Genome physical mapping with largeinsert bacterial clones by fingerprint analysis: Methodologies, source clone genome coverage and contig map quality. Genomics 84:941951.

Xu, Z., Van den Berg, M. A., Scheuring, C., Covaleda, L., Lu, H., Santos, F. A., Uhm, T., Lee, M. K., Wu, C., Liu, S. and Zhang, H. B. 2005. Genome physical mapping from large-insert clones by fingerprint analysis with capillary electrophoresis: A robust physical map of Penicillium chrysogenum. Nucleic Acids Res. 33:e50.

Zhang, H.-B. 2000. Construction and Manipulation of Large-Insert Bacterial Clone Libraries: Manual. Texas A\&M University, College Station, TX, U.S.A

\section{AUTHOR RECOMMENDED INTERNET RESOURCES}

United States Department of Energy Joint Genome Institute (JGI) genome sequence portal: genome.jgi-psf.org

Virginia Bioinformatics Institute microbial database: phytophthora.vbi.vt.edu

Arizona Genomics Computational Laboratory (AGCoL) P. sojae BAC physical map browser: phytophthora.vbi.vt.edu/webfpc/WebAGCoL/contig_bac/WebFPC

Oomycete Molecular Genetics Research Collaboration Network: pmgn.vbi.vt.edu

FPC (Fingerprinted Contigs) website: www.agcol.arizona.edu/software/fpc 